\title{
Análisis de Costos Nivelados de Electricidad de Plantas de Cogeneración usando Biomasa Forestal en el Departamento de Antioquia, Colombia
}

\author{
Juan D. Saldarriaga-Loaiza', Fernando Villada ${ }^{1}$ y Juan F. Pérez ${ }^{2}$ \\ Universidad de Antioquia, Facultad de Ingeniería, Grupo de Investigación GIMEL (1) Departamento de \\ Ingeniería Eléctrica, (2) Departamento de Ingeniería Mecánica, Calle 70 No. 52-21, Oficina 19-441, \\ Medellín-Colombia (e-mail: juand.saldarriaga@udea.edu.co; fernando.villada@udea.edu.co; \\ jfernando.perez@udea.edu.co)
}

Recibido May. 30, 2018; Aceptado Jul. 31, 2018; Versión final Sep. 13, 2018, Publicado Feb. 2019

\begin{abstract}
Resumen
En este trabajo se analizan los efectos de los incentivos tributarios de la Ley 1715 de 2014 (Ley colombiana de energías renovables) en el costo nivelado de electricidad (CNE) de plantas de generación de energía con biomasa forestal en el departamento de Antioquia. Con base en el potencial energético de la biomasa se consideraron las tecnologías de gasificación, combustión y ciclo orgánico Rankine (ORC). Se analizaron tres casos de estudio: 1) CNE sin incentivos, 2) CNE con incentivos y 3) CNE con incentivos, considerando una depreciación de activos a 10 años. Por otro lado, se realizó un análisis de sensibilidad para evaluar la variación en el CNE por cambios en el costo de la biomasa forestal, el costo de la tecnología de generación, la tasa mínima aceptable de rentabilidad y el periodo de gracia en los créditos para financiar las plantas. Finalmente, los resultados obtenidos muestran que el CNE del ORC $\left(13,2 \phi U \$ / k W_{\mathrm{e}} \mathrm{h}\right)$ es el que presenta mayor reducción $(11,2 \%)$, cuando se consideran los incentivos de la Ley 1715 de 2014 con depreciación de activos a 10 años, financiación del $50 \%$ de la inversión inicial y periodo de gracia a 5 años.
\end{abstract}

\section{Analysis of Levelized Costs of Electricity of Cogeneration Plants using Forest Biomass at the Antioquia Department, Colombia}

\begin{abstract}
In this paper the effects of the tax incentives of the Law 1715 of 2014 (Colombian law of renewable energies) on the levelized cost of electricity (LCOE) of cogeneration plants using forest biomass in the Antioquia Department are analyzed. Generation technologies such as Gasification, Combustion and Rankine Organic Cycle (ORC) were considered based on the energy potential of the forest biomass crops. Three study cases were carried out: 1) LCOE without tax incentives, 2) LCOE with tax incentives, and 3) LCOE with tax incentives considering ten years for assets depreciation. On the other hand, a sensitivity analysis was carried out to evaluate how the LCOE is affected due to changes in the forest biomass cost, the technology cost, the minimum acceptable return rate and the grace period on credits to finance the cogeneration plants. Finally, the results show that the LCOE with the ORC $(13,2 \mathrm{cU} \$ / \mathrm{kW} h)$ is the case with the greatest reduction $(11,2 \%)$, when the incentives of Law 1715 of 2014 with assets depreciation at 10 years, financing the $50 \%$ of the initial investment and grace period for 5 years are considered.
\end{abstract}




\section{INTRODUCCIÓN}

En los últimos años los sistemas de cogeneración se han convertido en una importante alternativa de generación distribuida para zonas rurales, en las cuales el suministro de energía es limitado e ineficiente (Caceres y Alca, 2016; Hallam y Contreras, 2015; Naqvi et al.,2017). Una de las principales ventajas de esta tecnología es la generación simultanea de energía eléctrica y térmica a partir de distintas fuentes de energía, entre las que se destacan la biomasa forestal, la biomasa residual y la biomasa orgánica de origen animal (Tosun, 2015). En la figura 1 se muestra una planta de cogeneración a pequeña escala de $120 \mathrm{~kW}$ e basada en gasificador de biomasa equicorriente acoplado a un grupo motor-generador.

Una planta de cogeneración puede ser utilizada para la producción de energía eléctrica y suministro de calor utilizable en procesos productivos (Oku et al., 2014) . Entre los beneficios obtenidos con esta tecnología se destacan: 1) respuesta a la demanda; 2) ventas de excedentes de energía al sistema interconectado nacional (SIN), definidas en las Leyes Colombianas 1215 de 2008 y 1715 de 2014, y el decreto 348 de 2017; 3) el aprovechamiento de la energía térmica para el calentamiento de agua, secado de madera y manejo de cultivos agrícolas, entre otros. La implementación de una planta de cogeneración implica un estudio de viabilidad técnico-financiero, el cual se puede realizar bajo diferentes metodologías y estudios, como el costo nivelado de electricidad (CNE) (Abdelhady et al., 2018; Castillo-Ramírez et al., 2015) y análisis termo-económicos (Chowdhury y Kibaara, 2016), respectivamente, o empleando herramientas computacionales como el Market Prices (Lupo y Kiprakis, 2016) y el HOMER (Renewable Energy Microgrid Software).

A partir del CNE, Uris et al. (2014) estudian un sistema de cogeneración a biomasa con ciclo orgánico Rankine para zonas rurales de España. El CNE calculado fue de 6,6 $\mathrm{cU} \$ / \mathrm{kW} \mathrm{eh}$, considerando una capacidad instalada de $2 \mathrm{MW}$, un factor de capacidad de 0,6, un precio de biomasa entre $(1,04-2,04 \$ \mathrm{U} \$ / \mathrm{kWh}$ th) y un periodo de retorno del proyecto de 10 años. You et al. (2017) evalúan la viabilidad técnica y financiera de un sistema de gasificación descentralizado, a partir del aprovechamiento de residuos de la palma de aceite, para suplir la demanda energética de las zonas rurales de Indonesia. EI CNE para este sistema fue de $46 \mathrm{cU} \$ / \mathrm{kW}$ eh, considerando un factor de capacidad de 1 y un total de 100 viviendas, cada una con un consumo diario de 0,4 kWeh. Hadidi y Omer (2017) analizan técnica y financieramente un sistema de gasificación y de digestión anaeróbica con residuos sólidos. El CNE para un sistema de gasificación de 75,62 MWe y de digestión anaeróbica de 12,2 MWe fue de 13 $\mathrm{U} \$ / \mathrm{kW}$ eh y 14 $\mathrm{UU} \$ / \mathrm{kW}$ eh, respectivamente.

Otros autores han propuesto incentivos económicos, variaciones en la tasa de interés y en la densidad poblacional para evaluar los cambios en los costos nivelados de electricidad. Es el caso de Chundawat y Rao (2016), quienes analizan dos plantas de biomasa para la generación de electricidad en zonas rurales de la India, con capacidades instaladas de 7,5 $\mathrm{MW}_{\mathrm{e}}$ y $8 \mathrm{MW}_{\mathrm{e}}$ conectadas a la red de distribución eléctrica. Los autores concluyen que el costo nivelado se ve esencialmente afectado por el aumento de la tasa de interés y la inflación. El aumento de estas tasas en una unidad implica un aumento aproximado del $10 \%$ en el CNE.

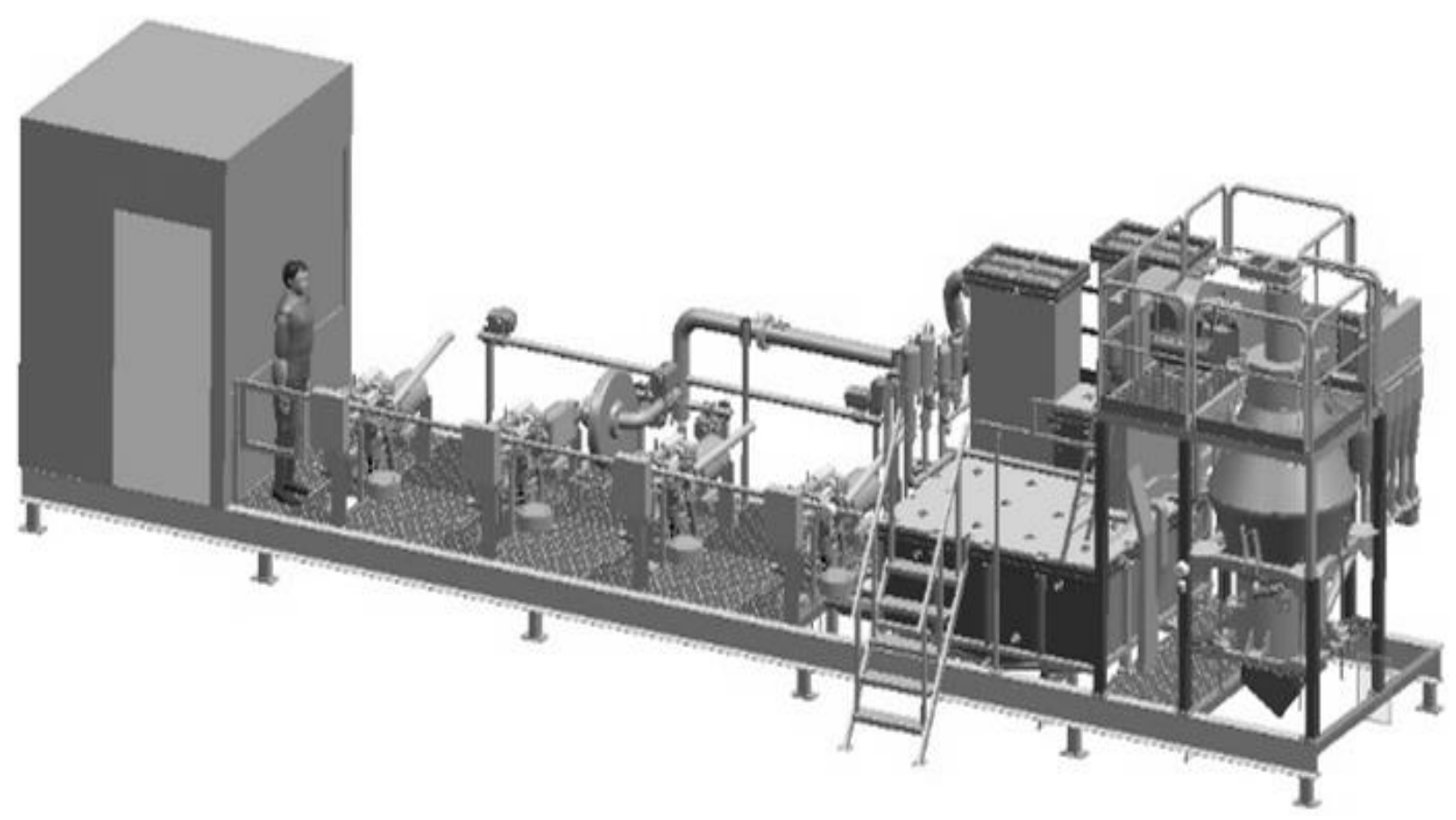

Fig. 1: Planta de cogeneración, adaptada de Pérez, Melgar y Tinaut (2014) 
En Colombia, según el plan de desarrollo para las fuentes no convencionales de energía (PDFNCE) año 2010, los costos nivelados de electricidad aplicados a zonas no interconectadas (ZNI) fueron aproximadamente de 9,5 cUSD/kWeh para gasificación, de 8,1 $\mathrm{cU} \$ / \mathrm{kW}$ h para biogás y de 7,7 $\mathrm{cU} \$ / \mathrm{kW}$ h para residuos sólidos. Para ese mismo periodo, el precio promedio de energía en bolsa fue de aproximadamente $4,5 \$ \cup \$ / \mathrm{kW}_{\mathrm{eh}}$ y para los ingenios azucareros, los cuales generan electricidad mediante bagazo de caña, el costo promedio de energía fue de 5,8 $₫ \cup \$ / k W$ h (Consorcio Energético Corpoema, 2010a). Castillo-Ramírez et al., (2015) calcularon los costos nivelados de distintas fuentes de energía; el CNE para la biomasa fue de $8,5 \mathrm{\phi U} \$ / \mathrm{KW}_{\mathrm{e}} \mathrm{h}$, considerando costos de inversión, costos fijos de operación y mantenimiento, precio de la biomasa, externalidades y una capacidad instalada de $40 \mathrm{MW}_{\mathrm{e}}$; por su parte, el CNE para una central hidroeléctrica de $800 \mathrm{MW}_{\mathrm{e}}$ fue de $5,5 \mathrm{\phi U} \$ / \mathrm{kW}$ h, siendo un $35 \%$ menor que el de biomasa.

Esta conclusión impulsa este estudio, teniendo en cuenta que Colombia genera alrededor del $60 \%$ al $70 \%$ de su electricidad con centrales hidroeléctricas, pero alrededor de 470.000 viviendas no cuentan con servicio de electricidad, de los cuales más del $70 \%$ se encuentran en zonas interconectables al Sistema Interconectado Nacional y $30 \%$ de ellos en zonas no interconectadas (Ministerio de Minas y Energía, 2015), no estando definido aún en el área rural el sistema más eficiente y económico para cubrir la demanda insatisfecha en las regiones de menor poder económico. Adicionalmente, después de 50 años de conflicto armado y con un entorno de posconflicto, la bioenergía tiene un impacto positivo en el contexto colombiano porque abre la posibilidad de modernizar la agricultura, generar oportunidades de empleo, mejorar la calidad de vida de las comunidades rurales, lograr el desarrollo sostenible con el medio ambiente y garantizar el suministro confiable de energía eléctrica y térmica (Gonzalez-Salazar et al., 2017; Montiel-Bohórquez et al., 2019). En este trabajo se proponen sistemas de cogeneración usando biomasa forestal, proveniente de plantaciones comerciales sostenibles, con el fin de energizar algunas zonas rurales del departamento de Antioquia. Por su relación costo-beneficio para la generación de energía, las especies forestales seleccionadas fueron Pinus Patula, Acacia Mangium y Eucalyptus sp (Osorio y Pérez, 2014) . Las tecnologías de aprovechamiento energético de la biomasa fueron seleccionadas en función del potencial de las plantaciones forestales comerciales de rápido crecimiento; las plantas se basan en gasificación, combustión y ciclo orgánico Rankine.

Los proyectos bioenergéticos basados en biomasa forestal sostenible (en este planteamiento no se considera biomasa a la selva húmeda tropical (pacifico Colombiano), Amazonas, ni bosques primarios ni secundarios) se analizan con los siguientes propósitos: 1) satisfacer la demanda energética de las zonas rurales con calidad, seguridad y confiabilidad; 2) generar electricidad con un costo competitivo frente a las fuentes convencionales de energía; 3) contribuir a la diversificación de la matriz energética del país; 4) reducir las emisiones de gases de efecto de invernadero con base en los acuerdos del COP $21 ; 5)$ lograr un desarrollo sostenible con menores impactos en el medio ambiente; 6) brindar alternativas de solución a los problemas de respuesta a la demanda e ineficiencia energética en las zonas rurales; 7) brindar seguridad energética al país; 8) generar desarrollo tecnológico y empleo local; 9) contribuir al desarrollo social para el posconflicto; 10) contribuir con el plan de acción indicativo PROURE 2017-2022 (Løtveit et al., 2014;Restrepo et al., 2016; Restrepo et al., 2018; Santamaría et al., 2014).

\section{METODOLOGÍA}

A continuación, se resume la metodología empleada para el cálculo y análisis de costos nivelados de electricidad para plantas de cogeneración a biomasa forestal. 1). Selección de la biomasa forestal existente más apropiada para las regiones del departamento de Antioquia con base en información secundaria silvicultural y energética; 2). Cálculo del potencial energético de las plantas de cogeneración a biomasa; 3). Selección de la tecnología de cogeneración más adecuada en función del potencial de la biomasa forestal seleccionada; 4). Estimación de los costos de generación de energía eléctrica de las plantas de cogeneración a biomasa, considerando los incentivos tributarios de la Ley 1715 de 2014.

\section{Potencial Energético}

En la tabla 1 se muestra el potencial energético de algunas plantaciones forestales comerciales de Antioquia registradas por el Instituto Colombiano Agropecuario (ICA) para el año 2014 (Instituto Colombiano Agropecuario, 2016). La potencia primaria (Pp), que se define como la cantidad de energía por unidad de tiempo que tienen disponible las plantas de cogeneración, se calcula mediante la ecuación 1 (Osorio y Pérez, 2014).

$$
P p[M W t h]=I M A \text { ha } \rho \operatorname{LHV} \frac{1 \text { año }}{8.765,8 h} \frac{1 h}{3.600 s} \frac{1}{1.000}
$$


Tabla 1: Potencial energético de biomasa forestal en Antioquia, año 2014

\begin{tabular}{|c|c|c|c|}
\hline Municipio & Especie & Hectáreas & Potencia primaria (MW th $^{\text {) }}$ \\
\hline \multirow{2}{*}{ Caldas } & Pinus Patula & 2281,22 & 11,83 \\
\cline { 2 - 4 } & Eucalyptus sp & 554,84 & 5,07 \\
\hline Maceo & Acacia Mangium & 8550,38 & 54,46 \\
\hline Entrerrios & Eucalyptus sp & 8926,32 & 81,59 \\
\hline Santa Rosa & Pinus Patula & 2976,63 & 15,44 \\
\hline Abejorral & Pinus Patula & 3378,55 & 17,53 \\
\hline El Retiro & Pinus Patula & 933,43 & 4,84 \\
\hline La Unión & Eucalyptus sp & 3866,46 & 35,34 \\
\hline Betania & Eucalyptus sp & 3969,78 & 36,28 \\
\hline Betulia & Pinus Patula & 375,62 & 1,95 \\
\hline Titiribí & Eucalyptus sp & 938,16 & 8,57 \\
\hline Total & & 36751,39 & 272,91 \\
\hline
\end{tabular}

Dónde el IMA es el incremento medio anual de las especies forestales, ha son las hectáreas del núcleo forestal, $\rho$ es la densidad en rollo de las especies forestales con un $20 \%$ de contenido de humedad y $L H V$ es el poder calorífico inferior de la biomasa forestal con un $20 \%$ de contenido de humedad. En la tabla 2 se muestra la información silvicultural y físico-química de las especies forestales seleccionadas (Osorio y Pérez, 2014; Pérez et al., 2017); dónde $L H V_{d s}$ es el poder calorífico en base seca de la biomasa forestal y $\rho_{b s}$ es la densidad en rollo en base seca de las especies forestales.

Tabla 2: Información silvicultural y fisicoquímica de las especies forestales seleccionadas

\begin{tabular}{|c|c|c|c|c|}
\hline Especie & $I M A\left(m^{3} /\right.$ ha año) & Turno (años) & $L H V_{b s}(\mathrm{~kJ} / \mathrm{kg})$ & $\rho_{\text {bs }}\left(\mathrm{kg} / \mathrm{m}^{3}\right)$ \\
\hline Pinus Pátula & 20 & 13 & 18.948 & 450 \\
\hline Acacia Mangium & 28 & 4 & 18.694 & 400 \\
\hline Eucalyptus sp & 25 & 7 & 18.948 & 650 \\
\hline
\end{tabular}

\section{Tecnologías de generación}

La generación simultanea de electricidad y energía térmica a partir del aprovechamiento de la biomasa forestal se puede llevar a cabo bajo diferentes tecnologías, tales como combustión, gasificación y ciclo orgánico Rankine. Vélez et al., (2012) definen que los sistemas de combustión con ciclo de vapor son ineficientes para potencias menores a $5 \mathrm{MW}$. Esto se debe a las altas presiones y temperaturas requeridas para un rendimiento óptimo, causando altos costos en operación y mantenimiento. Para potencias menores a $5 \mathrm{MW}_{\mathrm{e}}$, la gasificación presenta menores costos de inversión, operación y mantenimiento; mientras que para potencias mayores a $5 \mathrm{MW}$ e la combustión representa la mejor opción (Quuak et al., 1999).

El ciclo orgánico Rankine es viable técnica y financieramente para un rango de potencia entre 0,2 - $2 \mathrm{MW}_{\mathrm{e}}$; comparado con la gasificación, presenta menores costos por operación y mantenimiento (Vélez et al., 2012) y puede ser utilizada hasta una potencia neta eléctrica de 2,4 MWe (Obernberger y Thek, 2004). Así, La selección de la tecnología de generación dependerá de la energía eléctrica que se quiera suministrar. En la tabla 3 se presentan las tecnologías de generación con sus especificaciones de potencia eléctrica para el cálculo de costos nivelados de electricidad, con base en lo reportado en la literatura.

Tabla 3: Tecnologías de generación de energía

\begin{tabular}{|c|c|c|}
\hline Tecnología & $\begin{array}{c}\text { Potencia eléctrica neta }\left(M W_{e}\right) \\
\text { Ciclo Orgánico Rankine }\end{array}$ & $\begin{array}{c}\text { (Obernberger y Thek, 2004; Vélez } \\
\text { et al., 2012) }\end{array}$ \\
\hline Gasificación & $<5$ & (Quuak et al., 1999; Vélez et al., 2012) \\
\hline Combustión + Turbina a vapor & $>5$ & (Quuak et al., 1999; Vélez et al., 2012) \\
\hline
\end{tabular}




\section{Costo nivelado de electricidad}

EI CNE se define como el costo unitario de la electricidad generada, el cual contempla todos los costos del proyecto durante su vida útil. Por tal razón, define un precio constante al cual la energía debería ser vendida durante la vida operativa del proyecto. EI CNE considera la inversión inicial, los costos fijos y variables por operación y mantenimiento (OM), el costo del combustible y los incentivos tributarios (Bruck et al., 2018; Castillo-Ramírez et al., 2015). El CNE permite comparar los costos de generación entre diversas tecnologías, como solar fotovoltaica, eólica y biomasa, facilitando la selección de la tecnología con mayor viabilidad técnica y financiera. La ecuación del CNE presenta la ventaja de adaptarse a cambios en las estructuras técnicas y de costos de cada proyecto energético, permitiendo un análisis de sensibilidad en el costo de generación (Tran y Smith, 2018). El CNE se ha utilizado en los últimos años como herramienta financiera para calcular costos de generación y su uso se ha venido incrementando por la necesidad de evaluar energías alternativas, que permitan dar respuesta a la demanda en poblaciones con suministro de energía limitado e ineficiente (Caceres y Alca, 2016; Consorcio Energético Corpoema, 2010a; Hadidi y Omer, 2017; Osorio y Pérez, 2014. En las ecuaciones 2, 3 y 4 se muestran las expresiones para el CNE.

$$
C N E=C N E I+C N E V+C N E F+C N E C
$$

Dónde, CNEl: es la componente de inversión y se calcula como el cociente entre el costo anual uniforme equivalente (CAUE) y la generación anual de electricidad; CNEV: es la componente de costos variables por OM y se calcula como el cociente entre los costos variables anuales y la generación anual de electricidad; CNEF: es la componente de costos fijos por OM y se calcula como el cociente entre los costos fijos anuales y la generación anual de electricidad; CNEC: es la componente de costos por combustible y se calcula como el cociente entre los costos por combustible anuales y la generación anual de electricidad.

$$
C N E=\frac{I o+\sum_{t=1}^{n} \frac{C t}{(1+i)^{t}}}{\sum_{t=1}^{n} \frac{E t}{(1+i)^{t}}}
$$

Dónde, $l_{0}$ : es el costo de la inversión inicial, $C_{t}$ : son los costos operativos anuales, $E_{t}$ : es la cantidad de energía producida en un año, $i$ : es la tasa de descuento, $n$ : el tiempo de vida operacional en años, $t:$ la vida útil del proyecto.

\section{Ley Colombiana de Energías Renovables}

En el año 2014 el gobierno expidió la Ley de Energías Renovables 1715, la cual fomenta la inversión en proyectos de fuentes no convencionales de energía (FNCER) mediante 4 incentivos tributarios (Congreso de Colombia, 2014), los cuales se describen a continuación: 1). Los inversionistas pueden reducir anualmente de su renta, por los 5 años siguientes al año gravable en que hayan realizado la inversión, el $50 \%$ del valor total de la inversión realizada; 2). Exención del IVA a los equipos, elementos, maquinaria y servicios nacionales o importados que se destinen a la pre-inversión e inversión de FNCE; 3). Exención del pago de aranceles para los componentes anteriormente nombrados; 4). Depreciación acelerada de activos, la cual no será mayor al $20 \%$ anual como tasa global. El beneficio de los incentivos tributarios será mayor para aquellos proyectos de generación de energía que presenten un alto grado de inversión. Así el CAUE de la componente CNEI será menor y el CNE podría tener una reducción significativa. Por otro lado, la Ley 1715 de 2014 no impacta las componentes $C N E V+C N E F+C N E C$ porque la ley no estableció beneficios tributarios a la operación y mantenimiento de proyectos de energías renovables. Es importante anotar que la deducción de la inversión contemplada en el primer beneficio tiene la restricción de no poder superar el $50 \%$ de la renta líquida, tal como está explícito en la misma ley.

Para evaluar el efecto de los incentivos de la Ley 1715 de 2014 sobre el CNE, se aplica la expresión modificada de costos nivelados y el concepto de factor de impuestos $\Delta$, que se presentan en las ecuaciones 4 y 5 respectivamente (Villada et al., 2017).

$$
\begin{aligned}
& C N E=\Delta * C N E I+C N E V+C N E F+C N E C \\
& \Delta=\frac{1}{1-t}\left[1-t\left(\sum_{j=1}^{j=T 1} \frac{I j}{(1+i)^{j}}+\sum_{j=1}^{j=T 2} \frac{d j}{(1+i)^{j}}\right)\right]
\end{aligned}
$$

Donde, $t$ : es la tasa impositiva, $I j$ : es la tasa de recuperación de la inversión por reducción del impuesto a la 
renta, $T 1$ : es el número máximo de años permitidos para aplicar a la reducción del impuesto a la renta, $d j$ : es la tasa de depreciación acelerada y $T 2$ : es la vida útil de la instalación para propósitos de la depreciación acelerada.

\section{Casos de estudio}

Se realizaron tres casos de estudio para evaluar los efectos tributarios de la Ley 1715 de 2014 sobre los costos nivelados de electricidad de plantas de cogeneración a biomasa forestal en 2 núcleos forestales del departamento de Antioquia. En el primer caso, se calculó el CNE sin incentivos; en el segundo caso, se calculó el CNE con incentivos para determinar los efectos de la ley 1715 de 2014 en el CNE, y en el tercer caso, se calculó del CNE con incentivos tributarios con una depreciación de activos a 10 años, como alternativa para disminuir el CNE de las plantas de cogeneración. Los dos núcleos forestales seleccionados fueron los siguientes: 1.) Municipio de Caldas con 2.281 hectáreas de Pinus Pátula; 2). Municipio de Maceo con 8.850 hectáreas de Acacia Mangium. Según la tabla 3, para el primer núcleo se calcularon los costos nivelados de electricidad de dos tecnologías de generación: gasificación de lecho fijo equicorriente y ciclo orgánico Rankine. Para el segundo, se optó por la tecnología de ciclo de vapor (combustión más turbina de vapor). En la tabla 4 se detallan los datos técnicos y financieros de las tecnologías de generación con biomasa.

Tabla 4: Datos técnicos y financieros del sistema de Gasificación de lecho fijo equicorriente (Pérez et al., 2018)

\begin{tabular}{|c|c|c|c|c|c|c|c|c|}
\hline Tecnología & $\begin{array}{c}\text { Potencia } \\
\text { primaria } \\
\left(M W_{\text {th }}\right)\end{array}$ & $\begin{array}{c}\text { Eficiencia } \\
(\%)\end{array}$ & $\begin{array}{c}\text { Factor de } \\
\text { Capacidad }\end{array}$ & $\begin{array}{c}\text { Potencia } \\
\text { eléctrica } \\
\text { neta } \\
\left(M W_{e}\right)\end{array}$ & $\begin{array}{c}\text { Costo } \\
\text { Específico } \\
\left(U \$ / k W_{e}\right)\end{array}$ & $\begin{array}{c}\text { Costo por OM } \\
\text { variable } \\
\left(\phi U \$ / k W_{e} h\right)\end{array}$ & $\begin{array}{c}\text { Costo por } \\
\text { OM fijo } \\
\left(\phi U \$ / k W_{e} h\right)\end{array}$ & Referencias \\
\hline Gasificación & 11,83 & 19,5 & 0,94 & 2,3 & 2.466 & 0 & 1,50 & $\begin{array}{c}\text { (Pérez et al., } \\
2018)\end{array}$ \\
\hline ORC & 11,83 & 16,5 & 0,59 & 1,95 & 4.130 & 0 & 1,04 & $\begin{array}{c}\text { (Obernberger } \\
\text { y Thek, 2004) }\end{array}$ \\
\hline $\begin{array}{c}\text { Ciclo de } \\
\text { vapor }\end{array}$ & 54,46 & 15 & 0,91 & 8,12 & 1.657 & 0 & $0,83 \quad \begin{array}{c}\text { (Consorcio } \\
\text { Energético } \\
\text { Corpoema, } \\
2010 b ; \\
\text { et al., } 2017)\end{array}$ \\
\hline
\end{tabular}

Finalmente, en la tabla 5 se detallan datos complementarios tanto técnicos como financieros para el cálculo de los costos nivelados de electricidad de las plantas de cogeneración. La tasa de descuento se calculó mediante el costo promedio ponderado de capital (WACC, por sus siglas en inglés) y el precio de la biomasa forestal fue cotizado en diferentes industrias madereras de la ciudad de Medellín, tales como Tablemac, Duratex, y Madepatula, quienes cotizaron el costo de la biomasa para astillar. Los flujos de caja se trabajaron en dólares constantes del periodo actual asumiendo que todos los costos crecen a la tasa de inflación.

Tabla 5: Datos técnicos y financieros de los sistemas de cogeneración

\begin{tabular}{|l|c|}
\hline Descripción & Valor \\
\hline Inflación & $4 \%$ \\
\hline Tasa de descuento real & $8,1 \%$ \\
\hline Vida útil (años) & 20 \\
\hline Pinus Pátula (USD/ton) & 33,33 \\
\hline Acacia Mangium (USD/ton) & 33,33 \\
\hline
\end{tabular}

\section{RESULTADOS}

En la tabla 6 se muestran los resultados obtenidos para los casos de estudio y el efecto de los incentivos tributarios de la Ley 1715 de 2014 en el CNE de plantas de generación de energía con biomasa. Se observa que la gasificación es la tecnología que menor CNE presenta para una escala de generación de electricidad cercana a los $2.000 \mathrm{~kW}$. Esto se debe a que tiene mayor eficiencia, mayor factor de capacidad y menor costo específico con respecto a la tecnología de ciclo orgánico Rankine, según la tabla 4.

EI CNE de cada tecnología disminuye cuando los incentivos tributarios de la Ley 1715 de 2014 son aplicados, siempre y cuando se considere una depreciación de activos a 10 años, ya que el efecto de la tasa de impuesto de renta (tasa impositiva) es menor cuando en el flujo de caja se presentan deducciones por depreciación de activos durante un mayor periodo de tiempo (10 años). Las tecnologías con mayor costo específico presentan 
una mayor reducción en el CNE, como es el caso del ciclo orgánico Rankine, que presenta una reducción del $1,75 \%$ porque los incentivos de la Ley 1715 de 2014 solo afectan la componente de inversión del CNE, según la ecuación 5. La tecnología de ciclo de vapor (combustión más turbina a vapor) es la que presenta menor porcentaje de reducción en el CNE por tener el menor costo específico.

Tabla 6: CNE para las plantas de cogeneración, casos 1,2 y 3 sin financiación

\begin{tabular}{|c|c|c|c|c|c|}
\hline Tecnología & $\begin{array}{c}\text { Potencia neta } \\
\text { eléctrica }(\mathrm{kWe})\end{array}$ & $\begin{array}{c}\text { CNE }(\phi \cup \$ / \mathrm{kW} h) \\
\text { Caso } 1\end{array}$ & $\begin{array}{c}\text { CNE con } \\
\text { incentivos } \\
\left(\phi U \$ / k W_{e} h\right) \\
\text { Caso 2 }\end{array}$ & $\begin{array}{c}\text { CNE con } \\
\text { incentivos } \\
\left(\phi U \$ / k W_{e} h\right) \\
\text { Caso 3 }\end{array}$ & $\begin{array}{c}\text { Reducción } \\
\text { máxima en el } \\
\text { CNE }\end{array}$ \\
\hline $\begin{array}{c}\text { Ciclo Orgánico } \\
\text { Rankine }\end{array}$ & 1.950 & 14,87 & 15,06 & 14,61 & $1,75 \%$ \\
\hline Gasificación & 2.300 & 8,45 & 8,52 & 8,35 & $1,2 \%$ \\
\hline Ciclo de vapor & 8.120 & 7,65 & 7,7 & 7,58 & $0,92 \%$ \\
\hline
\end{tabular}

Comparando los resultados de los casos 1 y 2 se observa que los incentivos tributarios de la Ley 1715 de 2014 generan un incremento en el CNE cuando se considera una depreciación acelerada de activos a 5 años, ya que los ingresos del proyecto por concepto de venta de energía son inferiores a la suma de los egresos (OM y combustible) y a la depreciación anual de los activos; caso contario sucede cuando se considera una depreciación acelerada de activos a 10 años, en el cual el CNE si presenta un porcentaje de reducción porque el efecto de la tasa impositiva es menor. Así, con una depreciación acelerada de activos a 5 años no se tendría el beneficio de descuento en impuesto de renta al tener una renta líquida negativa y los incentivos tributarios de la ley 1715 de 2014 solo podrían ser aprovechados a plenitud por empresas mayores con otros ingresos diferentes a los generados por proyectos de energías renovables, los cuales les permiten obtener deducciones por renta líquida positiva (Castillo-Ramírez et al., 2015; Villada et al., 2017) .

\section{ANÁLISIS DE SENSIBILIDAD}

En esta sección se analiza la variación del CNE obtenido en el caso de estudio número 2 en función de los cambios en el costo de la biomasa forestal, el costo específico de la tecnología de generación, la tasa mínima aceptable de rentabilidad (TMAR), el período de gracia en los créditos de financiación de las plantas y el tiempo de depreciación de los activos. En el caso 2, se calculó el CNE para cada tecnología de generación, considerando los incentivos tributarios de la ley 1715 de 2014; y fue seleccionado como caso base para evaluar el impacto de los incentivos tributarios sobre cambios en las componentes del CNE, según las ecuaciones 4 y 5 . En las figuras 2, 3, 4, 5 y 6 se presentan los resultados obtenidos para el análisis de sensibilidad.

\section{Efecto del costo de la biomasa forestal}

Una variación en la componente de costos por combustible produce un incremento o reducción en el CNE, según la ecuación 4 . El incremento en el costo del combustible puede darse por el aumento en el costo de la tonelada de biomasa y su acondicionamiento, por la ineficiencia del sistema de cogeneración (mayor consumo de biomasa), por menor poder calorífico de la biomasa, entre otros. Según la figura 2, el CNE de cada tecnología aumenta con el incremento del costo de la tonelada de biomasa forestal, considerando una variación del costo de la biomasa entre 26 y 40 (USD/ton). Para un precio de biomasa de 40 USD/ton, el cual representa un incremento aproximado del $20 \%$ del precio cotizado (tabla 5), las tecnologías ORC, gasificación y ciclo de vapor presentan un CNE de 15,83 $\mathrm{\omega} \mathrm{U} \$ / \mathrm{kW}$ h, 9,22 $\mathrm{\phi U} \$ / \mathrm{kW}$ h y 8,54 $\mathrm{\phi U} \$ / \mathrm{kW}$ h respectivamente, produciéndose un incremento del $5,11 \%, 8,22 \%$ y $10,9 \%$ con respecto a los resultados del caso 2 (tabla 6 ). La mayor variación en el CNE se presenta en aquellas tecnologías que tienen menores costos específicos, ya que al tener componentes de inversión baja (CNEI), el aumento en la componente de costos por combustible $(C N E C)$ causa un mayor efecto en el CNE. Así, el ORC y el ciclo de vapor presentan la menor y mayor variación por ser las tecnologías con el mayor y menor costo específico respectivamente (ver tabla 4). Se destaca que los efectos de la Ley 1715 de 2014 permanecen constantes ante la variación del costo de la biomasa, ya que estos solo afectan la componente de inversión del CNE, según la ecuación 4.

\section{Efecto del costo específico de la tecnología de generación}

Una variación en la componente de inversión (CNEI) produce un incremento o reducción en el CNE, según la ecuación 4. El incremento en el costo específico de las tecnologías puede darse por el aumento en el costo del equipamiento, las obras civiles, la subestación entre otros. En la figura 3 , se muestra el comportamiento 
del CNE de cada tecnología cuando el costo específico se incrementa y reduce hasta un $20 \%$ y $-20 \%$ respectivamente. El incremento del costo específico causa un aumento en la componente de inversión del CNE (ecuación 4), y por tal razón, el CNE tiende a ser mayor. De las tres tecnologías, el ORC es la tecnología que mayor sensibilidad presenta por tener mayor costo específico. Finalmente, comparando las figuras 2 y 3 , se observa que el CNE de las tecnologías con menor costo específico presenta mayor sensibilidad a la variación del costo de la biomasa que a la variación del costo específico.

\section{Efecto de la tasa mínima aceptable de rentabilidad (TMAR)}

La TMAR se define como el costo de oportunidad que un inversionista espera obtener con su inversión a una tasa mínima de ganancia. La TMAR se tiene en cuenta para el cálculo de la tasa de descuento e implícitamente se encuentra en el CNE de cada tecnología. En la figura 4, se muestra la variación del CNE en función de la TMAR, la cual toma valores entre el $6 \%$ y $14 \%$ efectivo anual. Para una TMAR del $14 \%$, las tecnologías ORC, gasificación y ciclo de vapor presentan un CNE de 15,87 фU $\$ / \mathrm{kW}$ eh, 8,825 $\mathrm{\phi U} \$ / \mathrm{kW}$ eh y $7,913 \pitchfork \mathrm{U} \$ / \mathrm{kW}$ h respectivamente, los cuales representan un incremento del $5,4 \%, 3,6 \%$ y $2,8 \%$ respectivamente, con respecto a los resultados del caso 2 . El incremento del CNE en función de la TMAR se debe a que la electricidad debe ser vendida a un mayor precio para satisfacer la rentabilidad mínima del inversionista, y el efecto es mayor para aquellas tecnologías que presentan mayor costo específico. Así, el CNE del ORC es el que presenta mayor variación frente a la TMAR.

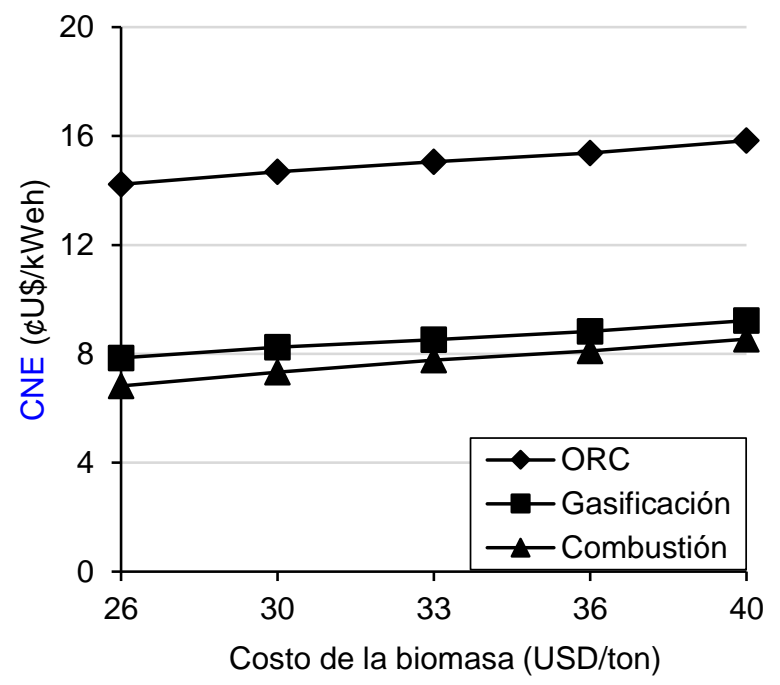

Fig. 2: Efecto del costo de la biomasa forestal

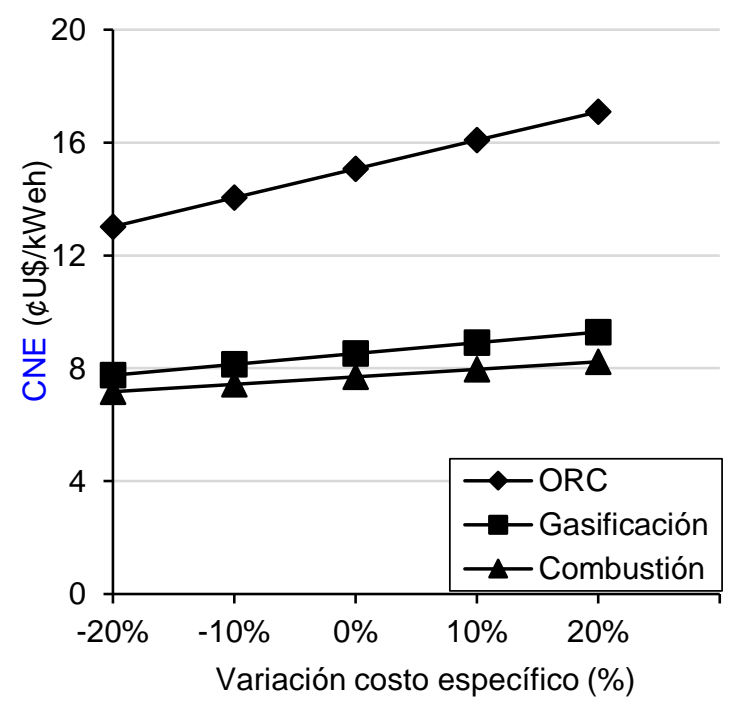

Fig. 3: Efecto del costo específico

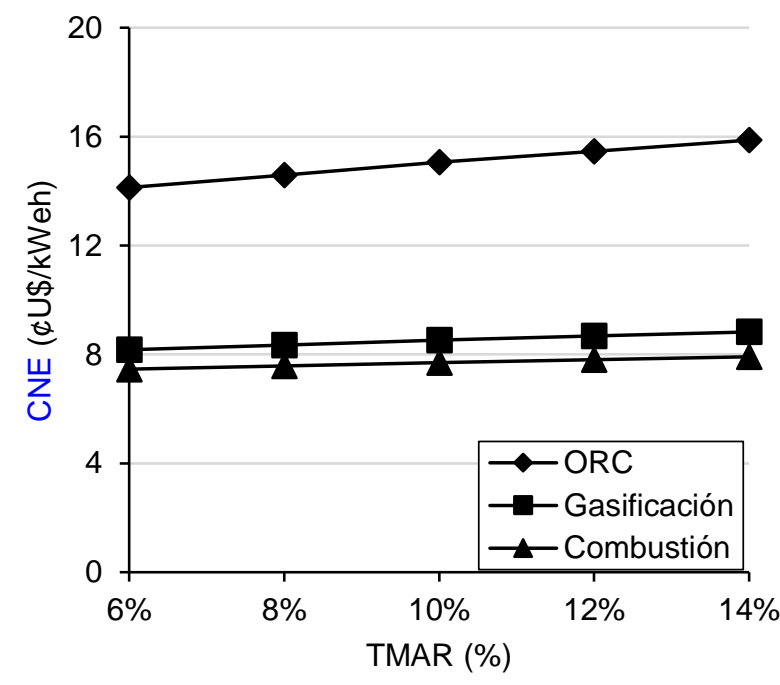

Fig. 4: Efecto de la TMAR

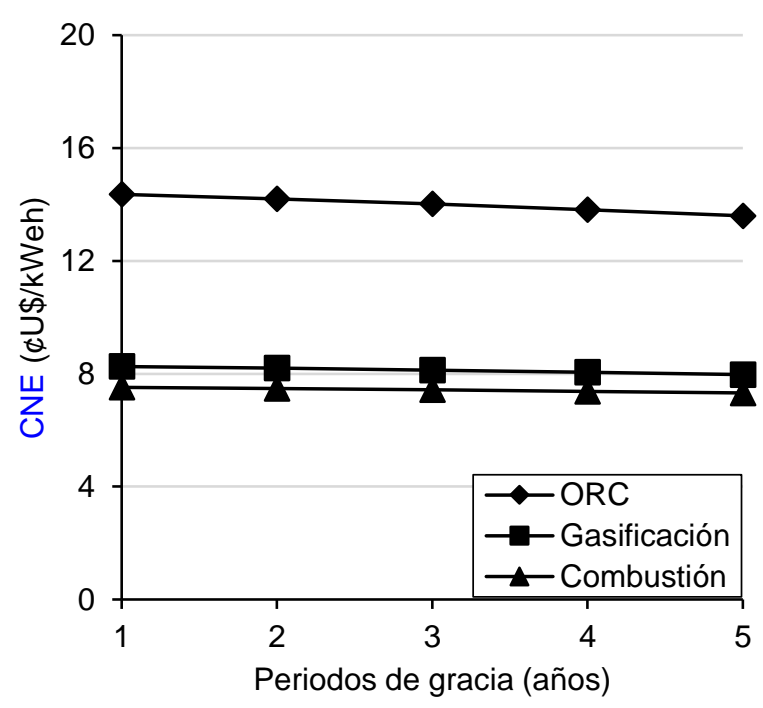

Fig. 5: Efecto de los períodos de gracia 


\section{Efecto de los períodos de gracia en créditos para financiar las plantas}

El periodo de gracia en un crédito permite aplazar el pago de las cuotas hasta cierto periodo de tiempo. En la figura 5 , se muestra la variación del CNE del caso 2, que fue calculado sin financiación, en función de los años de gracia, considerando para cada tecnología un crédito del $50 \%$ de la inversión inicial y un periodo de gracia que va desde 1 hasta 5 años. Los resultados obtenidos se comparan con los del caso 1, que fue evaluado sin financiación. Según la figura 5, para un periodo de gracia de 1 año, las tecnologías ORC, gasificación y ciclo de vapor presentan un CNE de 14,36 $\mathrm{\phi U} \$ / \mathrm{kW}_{\mathrm{e}} \mathrm{h}, 8,26 \mathrm{\phi U} \$ / \mathrm{kW}$ eh y 7,5 $\mathrm{\phi U} \$ / \mathrm{kW}$ eh respectivamente, produciéndose una reducción del 3,4\%,2,2\% y $2 \%$ con respecto a los resultados del caso 1; y para un periodo de gracia de 5 años, presentan un CNE de 13,6 $\mathbf{\phi U} \$ / \mathrm{kW}$ h, 7,97 $\mathrm{\phi U} \$ / \mathrm{kW}$ eh y 7,32 фU $\$ / \mathrm{kW}$ eh respectivamente, debido a una reducción del $8,5 \%, 5,7 \%$ y $4,3 \%$ con respecto a los resultados del caso 1. Esta tendencia de reducción se debe a que el efecto del impuesto de renta (tasa impositiva) sobre el valor presente neto es menor para aquellas plantas de cogeneración que cuentan con financiación y con mayor periodo de gracia; así, la electricidad producida por cada planta podría ser vendida a un menor precio.

Es importante mencionar que el efecto del impuesto de renta tiende a ser menor por las siguientes razones: 1) la depreciación acelerada a 5 años causa una renta líquida gravable negativa durante los primeros 5 años de operación de las plantas, generando un impuesto de renta cero; 2) después del año 5 la renta líquida gravable es positiva pero tiende a reducirse cuando el periodo de gracia aumenta, ya que los gastos financieros (pago de intereses de los créditos), que no se pagaron durante el periodo de gracia, reducen el valor de la renta líquida gravable, causando que el impuesto de renta disminuya. Por tales razones, la depreciación acelerada y el pago de intereses con mayor periodo de gracia generan una mayor variación en el CNE de tecnologías con mayor costo específico, como es el caso del ORC. Finalmente, bajo este escenario de estudio tampoco se pudo aprovechar el incentivo de la reducción del $50 \%$ de la inversión inicial en 5 años, mediante el impuesto de renta, ya que la renta líquida gravable fue negativa durante los primeros 5 años, tal como sucedió en el caso 2.

\section{Efecto de los periodos de gracia en créditos para financiar las plantas}

En el escenario previo (efecto de los períodos de gracia en créditos para financiar las plantas) se determinó que la financiación del $50 \%$ de la inversión inicial, con un periodo de gracia de 5 años y con los incentivos de la Ley 1715 de 2014, reduce el CNE hasta en un 8,5\% para el ORC con respecto al CNE del caso 1; caso contrario para el caso 2, que considerando los incentivos de la Ley 1715 de 2014 pero sin financiación, el CNE del ORC se aumenta en un 1,3\%, con respecto al CNE del caso 1. Así, para determinar cómo se pude reducir en mayor porcentaje el CNE, se tomó el escenario previo y se modificó el periodo de depreciación de activos de 5 a 10 años. Esto con el objetivo de evaluar viabilidad financiera de este tipo de proyectos en el marco de la legislación actual.

En la figura 6, se muestra la variación del CNE de cada tecnología en función de los años de gracia, considerando un crédito del $50 \%$ de la inversión inicial, un periodo de gracia que va desde 1 hasta 5 años y los beneficios de la Ley 1715 de 2014 con depreciación de activos a 10 años.

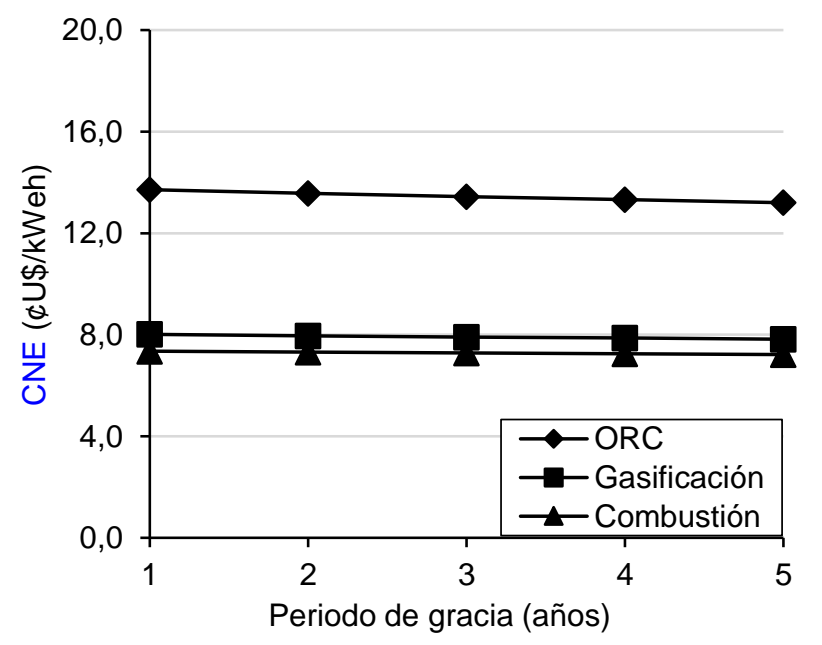

Fig. 6: Efecto de los períodos de gracia con depreciación a 10 años 
Los resultados de la figura 6 muestran que, para un periodo de gracia de 1 año, las tecnologías ORC,

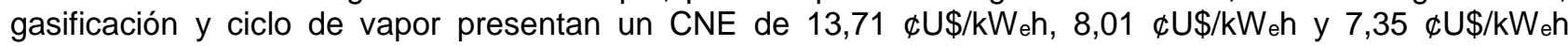
respectivamente. Esta condición del proyecto deriva una reducción del 7,8\%, 5,2\% y 3,9\% con respecto a los resultados del caso 1; para un periodo de gracia de 5 años, presentan un CNE de 13,2 USD/kW eh, 7,8 $\$ U \$ / W_{\text {eh y }} 7,2 \$ U \$ / \mathrm{kW}_{\text {e }}$ respectivamente, con una reducción del $11,2 \%, 7,7 \%$ y $5,9 \%$ con respecto a los resultados del caso 1. Estas reducciones son mayores que las presentadas en el análisis de la figura 5 , por lo que contar con incentivos que consideren una depreciación de activos a 10 años y un periodo de gracia de 5 años, favorecería el CNE de aquellos proyectos de generación de energía que cuenten con ingresos bajos y serían competitivos frente a otras fuentes de energía renovables, como solar fotovoltaica $(20 \mathrm{MW}$ ) , la cual presenta un costo de generación de 22 \$U $\$ / k W_{\text {eh }}$ (Castillo-Ramírez et al., 2015).

\section{CONCLUSIONES}

En este artículo se presentó el cálculo del CNE para tres sistemas de cogeneración con biomasa forestal, considerando los incentivos tributarios de la Ley 1715 de 2014. Esta metodología puede ser utilizada para calcular el CNE de otras FNCER.

Los efectos de la Ley 1715 de 2014 son más notorios para aquellas tecnologías que presentan altos costos específicos, ya que los incentivos solo impactan la componente de inversión del CNE. La tecnología ORC fue la que presentó la mayor reducción en el CNE por tener mayor costo específico. Esta reducción fue de aproximadamente del $11,2 \%$, la cual consideró incentivos tributarios con depreciación de activos a 10 años, financiación del $50 \%$ de la inversión inicial y periodo de gracia a 5 años.

Los costos nivelados de las tecnologías presentaron mayor sensibilidad a las variaciones del costo por combustible, aunque el ORC por tener un alto costo específico, también presentó sensibilidad a las variaciones del costo asociado a la tecnología. Por otro lado, es preferible que las pequeñas empresas cuenten con financiación, depreciación a 10 años y periodo de gracia de 5 años, para aprovechar los beneficios de la Ley 1715 de 2014 y así poder reducir el costo de electricidad en 13,2 $\$ \cup \$ / k W$ eh para el ORC con potencia de $1.950 \mathrm{~kW}$, en 7,8 $₫ \mathrm{U} \$ / \mathrm{kW}$ eh para la gasificación con $2.300 \mathrm{~kW}$ e de potencia y en 7,2 $\pitchfork \cup \$ / k W$ eh para la combustión con una potencia de 8.120 kWe

Según este trabajo, para la generación de electricidad con 2.281 hectáreas de Pinus Pátula, en el municipio de Caldas, es mejor utilizar tecnología de gasificación que el ORC por presentar un menor CNE y un mayor factor de capacidad. Para el municipio de Maceo, con 8.850 hectáreas de Acacia Mangium, la combustión con ciclo de vapor fue la tecnología técnica y financieramente más adecuada para potencial energético disponible. Comparando el CNE de cada tecnología con otras fuentes de energía como solar fotovoltaica (20 $\mathrm{MW}_{\mathrm{e}}$ ), con costo de generación de $22 \mathrm{\phi U} \$ / \mathrm{kW}_{\mathrm{e}}$ h respectivamente, se concluye que los proyectos de generación de electricidad con biomasa forestal son viables técnica y financieramente en Colombia.

Los proyectos de generación de energía mediante el aprovechamiento de la biomasa forestal en zonas rurales podrían contribuir al desarrollo de los acuerdos logrados para el posconflicto, ya que el gobierno se comprometió a ampliar la cobertura eléctrica, la promoción y aplicación de soluciones tecnologías apropiadas de generación de energía, capacitación en el uso adecuado de la energía, etc. Así, estas alternativas de generación de energía representan proyectos productivos en el marco del posconflicto.

\section{REFERENCIAS}

Abdelhady, S., D. Borello y A. Shaban, Techno-Economic Assessment of Biomass Power Plant Fed with Rice Straw: Sensitivity and Parametric Analysis of the Performance and the LCOE, doi.org/10.1016/j.renene.2017.09.040, Renewable Energy, 115, 1026-1034 (2018)

Bruck, M., P. Sandborn, y N. Goudarzi, A Levelized Cost of Energy (LCOE) Model for Wind Farms that Include Power Purchase Agreements (PPAs), doi.org/10.1016/j.renene.2017.12.100, Renewable Energy, 122, 131-139 (2018)

Caceres, E. y J.J. Alca, Rural Electrification Using Gasification Technology: Experiences and Perspectives, doi:10.1109/TLA.2016.7587637, IEEE Latin America Transactions, 14(7), 3322-3328 (2016)

Castillo-Ramírez, A., D. Mejía-Giraldo y J.D. Giraldo-Ocampo, Geospatial Levelized Cost of Energy in Colombia: GeoLCOE, Innovative Smart Grid Technologies Latin America (ISGT LATAM) 2015 IEEE PES, 298-303, MontevideoUruguay, 5-7 de Octubre (2015)

Castillo-Ramírez, A., D. Mejía-Giraldo y J. D. Molina-Castro, Fiscal Incentives Impact for RETs Investments in Colombia, doi.org/10.1080/15567249.2016.1276648, Energy Sources, Part B: Economics, Planning, and Policy, 0(0), 1-6 (2017)

Chowdhury, S. y S. K. Kibaara, Review of Economic Modelling for Quantifying the Environmental Impacts of Renewable Energy Sources, IEEE PES Power Africa, 280-284, Livingstone-Zambia, 28 de Junio-03 de Julio (2016) 
Chundawat, S.S. y K.V. Rao, Levelized Electricity Cost of Two Grid Connected Biomass Power Plants, Biennial International Conference on Power and Energy Systems: Towards Sustainable Energy (PESTSE), 1-6, Bangalore-India, 21-23 de Enero (2016)

Congreso de Colombia, Ley 1715 de 2014, 1-26, Bogotá, Colombia (2014)

Consorcio Energético Corpoema, Formulación de un Plan de Desarrollo para las Fuentes no Convencionales de Energía en Colombia (PDFNCE) V1, UPME, Colombia (2010)

Consorcio Energético Corpoema, Formulación de un Plan de Desarrollo para las Fuentes no Convencionales de Energía en Colombia (PDFNCE) V3, UPME, Colombia (2010)

Gonzalez-Salazar, M. A., M. Venturini y otros tres autores, Combining an Accelerated Deployment of Bioenergy and Land Use Strategies: Review and Insights for a Post-Conflict Scenario in Colombia, doi:10.1016/j.rser.2017.01.082, Renewable and Sustainable Energy Reviews, 73, 159-177 (2017)

Hadidi, L. A. y M. M. Omer, A Financial Feasibility Model of Gasification and Anaerobic Digestion Waste-to-Energy (WTE) Plants in Saudi Arabia, doi:10.1016/j.wasman.2016.09.030, Waste Management, 59, 90-101 (2017)

Hallam, C.R.A. y C. Contreras, Evaluation of the Levelized Cost of Energy Method for Analyzing Renewable Energy Systems: A Case Study of System Equivalency Crossover Points Under Varying Analysis Assumptions, doi:10.1109/JSYST.2013.2290339, IEEE Systems Journal, 9(1), 199-208 (2015)

Instituto Colombiano Agropecuario, Plantaciones Forestales Comerciales Registradas para Antioquia, Gobernación de Antioquia, Colombia (2016)

Løtveit, M., J. A. Suul, E. Tedeschi y M. Molinas, A Study of Biomass in a Hybrid Stand-Alone Micro-Grid for the Rural Village of Wawashang Nicaragua, Ninth International Conference on Ecological Vehicles and Renewable Energies (EVER), 1-7, Montecarlo-Monaco, 25-27 de Marzo (2014)

Lupo, S. y A. E. Kiprakis, The Impact of Renewable Energy Resources on the Electricity Prices of the United Kingdom, 13th International Conference on the European Energy Market (EEM),1-5, Porto-Portugal, 6-9 de Junio (2016)

Ministerio de Minas y Energía, Decreto 1623 de 2015, 1-15, Bogotá, Colombia (2015)

Montiel-Bohórquez, N.D y J.F. Pérez, Generación de Energía a partir de Residuos Sólidos Urbanos: Estrategias Termodinámicas para Optimizar el Desempeño de Centrales Térmicas, Información Tecnológica, 30(1), en prensa (2019)

Naqvi, M., J. Yan, E. Dahlquist y S. R. Naqvi, Off-Grid Electricity Generation using Mixed Biomass Compost: A Scenariobased Study with Sensitivity Analysis, doi.org/10.1016/j.apenergy.2017.02.005, Applied Energy, 201, 363-370 (2017)

Obernberger, I. y G. Thek, Techno-Economic Evaluation of Selected Decentralized CHP Applications based on Biomass Combustion with Steam Turbine and ORC Processes, IEA Bioenergy, Austria (2004)

Oku, M., T. Sakoda, N. Hayashi y D. Tashima, Basic Characteristics of a Heat and Electricity Combined Generation System using Biomass Fuel, International Conference on Renewable Energy Research and Application (ICRERA), 222-228, WIUSA, 19-22 de Octubre (2014)

Osorio, L.F. y J. F. Pérez, Biomasa Forestal como Alternativa Energética: Análisis Silvicultural, Técnico y Financiero de

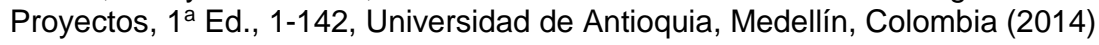

Pérez, J.F., A. Melgar y F.V. Tinaut, Modeling of Fixed Bed Downdraft Biomass Gasification: Application on Lab-Scale and Industrial Reactors, doi:10.1002/er.3045, International Journal of Energy Research, 38(3), 319-338 (2014)

Pérez, J.F., L. Osorio y A. Agudelo, A Technical-Economic Analysis of Wood Gasification for Decentralized Power Generation in Colombian Forest Cores, International Journal of Renewable Energy Research (IJRER), ISSN:1309-0127, 8(2), 1071-1084 (2018)

Pérez, J.F., M.R. Pelaez-Samaniego y M. Garcia-Perez, Torrefaction of Fast-Growing Colombian Wood Species, doi: 10.1007/s12649-017-0164-y, Waste and Biomass Valorization, 1-13 (2017)

Quuak P., H. Knoe y H. Stassen, Energy from Biomass: A review of Combustion and Gasification Technologies, World Bank Technical, 422, 1-99 (1999)

Restrepo, A.R., D.F. Manotas y C.A. Lozano, Self-Generation of Electricity, Assessment and Optimization under the New Support Schemes in Colombia, doi.org/10.1109/TLA.2016.7459614, IEEE Latin America Transactions, 14(3), 1308-1314 (2016)

Restrepo, A.R., S.E. Nope y D.E. Enríquez, Beneficios Económicos de la Gestión de la Demanda y la Energía Autogenerada en el Contexto de la Regulación Colombiana, doi:10.4067/S0718-07642018000100105, Información Tecnológica, 29(1), 105-106 (2018)

Santamaria, F., J. Hernandez y C.L Trujillo, Regulation on Distributed Generation: Latin American Case, IEEE PES Transmission Distribution Conference and Exposition - Latin America (PES T D-LA), 1-7, Medellín- Colombia, 10-13 de Septiembre (2014)

Tran, T.T.D. y A.D. Smith, Incorporating Performance-Based Global Sensitivity and Uncertainty Analysis into LCOE Calculations for Emerging Renewable Energy Technologies, doi.org/10.1016/j.apenergy.2018.02.024, Applied Energy, 216, 157-171(2018) 
Tosun, Y. I., 5MW Hybrid Power Generation with Agriculture and Forestry Biomass Waste Co-Incineration in Stoker and Subsequent Solar Panel (CSP) ORC Station, International Conference on Renewable Energy Research and Applications (ICRERA), 583-589, Palermo-Italia, 22-25 de Noviembre (2015)

Uris, M., J. I. Linares y E. Arenas, Techno-Economic Feasibility Assessment of a Biomass Cogeneration Plant Based on an Organic Rankine Cycle, doi: 10.1016/j.renene.2014.01.022, Renewable Energy, 66(C), 707-713 (2014)

Vélez, F., J.J. Segovia y otros cuatro autores, A Technical, Economical and Market Review of Organic Rankine Cycles for the Conversion of Low-Grade Heat for Power Generation, doi:10.1016/j.rser.2012.03.022, Renewable and Sustainable Energy Reviews, 16(6), 4175-4189 (2012)

Villada, F., J.M. Lezama y N. Muñoz, Effects of Incentives for Renewable Energy in Colombia, doi:10.11144/Javeriana.iyu21-2.eire, Ingeniería y Universidad, 21(2), 257-272 (2017)

You, S., H. Tong y otros tres autores, Techno-Economic and Greenhouse Gas Savings Assessment of Decentralized Biomass Gasification for Electrifying the Rural Areas of Indonesia, doi:10.1016/j.apenergy.2017.10.001, Applied Energy, 208, 495-510 (2017) 